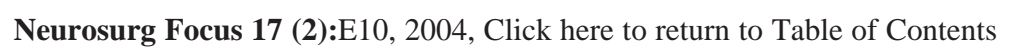

\title{
Stereotactic radiosurgery in patients with glomus jugulare tumors
}

\author{
Bruce E. Pollock, M.D. \\ Department of Neurologic Surgery, and Division of Radiation Oncology, Mayo Clinic College of \\ Medicine, Rochester, Minnesota
}

\begin{abstract}
Object. Microsurgical removal of glomus jugulare tumors is frequently associated with injury of the lower cranial nerves. To decrease the morbidity associated with tumor management in these patients, gamma knife surgery (GKS) was performed as an alternative to resection.

Methods. Between 1990 and 2003, 42 patients underwent GKS as the primary management (19 patients) or for recurrent glomus jugulare tumors (23 patients). Facial weakness and deafness were more common in patients with recurrent tumors than in those in whom primary GKS was performed $(48 \%$ compared with $11 \%, \mathrm{p}=0.02)$. The mean tumor volume was $13.2 \mathrm{~cm}^{3}$; the mean tumor margin dose was $14.9 \mathrm{~Gy}$. The mean follow-up period for the 39 patients in whom evaluation was possible was 44 months (range 6-149 months). After GKS, 12 tumors (31\%) decreased in size, $26(67 \%)$ were unchanged, and one $(2 \%)$ grew. The patient whose tumor grew underwent repeated GKS. Progression-free survival after GKS was $100 \%$ at 3 and 7 years, and $75 \%$ at 10 years. Six patients (15\%) experienced new deficits (hearing loss alone in three, facial numbness and hearing loss in one, vocal cord paralysis and hearing loss in one, and temporary imbalance and/or vertigo in one). In 26 patients in whom hearing could be tested before GKS, hearing preservation was achieved in 86 and $81 \%$ at 1 and 4 years posttreatment, respectively. No patient suffered a new lower cranial nerve deficit after one GKS session; the patient in whom repeated GKS was performed experienced a new vocal cord paralysis 1 year after his second procedure.

Conclusions. Gamma knife surgery provided tumor control with a low risk of new cranial nerve injury in early follow-up review. This procedure can be safely used as a primary management tool in patients with glomus jugulare tumors that do not have significant cervical extension, or in patients with recurrent tumors in this location.
\end{abstract}

\section{KEY WORDS • glomus jugulare tumor • jugular foramen • gamma knife surgery}

Glomus jugulare tumors arise from the paraganglia of the chemoreceptor system and occur in intra- and extracranial locations. Local invasion of adjacent structures or the effect of the tumor mass causes symptoms. Neurovascular structures within the hypoglossal canal, jugular foramen, and temporal bone can be affected. Treatment has included resection, embolization, external-beam radiation therapy, or a combination of these modalities. ${ }^{6}$

Surgery has been performed most frequently in young patients or in those with complete loss of cranial nerve function. Complete surgical removal is not always possible; gross-total resection has been accomplished in 40 to $80 \%$ of cases in reported series ${ }^{5,6}$ External-beam radiation therapy has been used in elderly patients and for symptomatic tumors that were considered unresectable, were incompletely removed, or had recurred after resection. $2,9,15,16$ Control of glomus jugulare tumors after external-beam radiation therapy has ranged from 85 to $100 \%$, with complication rates of 0 to $10 \%$. Complications associated with external-beam radiation therapy have included

Abbreviations used in this paper: GKS = gamma knife surgery; $\mathrm{MR}=$ magnetic resonance. necrosis of the temporal bone or brain, mastoiditis, and other local tissue injury.

As an alternative to resection or external-beam radiation therapy, radiosurgery has been used to manage disease in patients with glomus jugulare tumors in the hope of achieving high tumor control rates and symptom response similar to those seen with external-beam radiation therapy. ${ }^{3,4,7,11}$ In this paper we describe our experience with GKS in glomus jugulare tumors over the past 15 years.

\section{CLINICAL MATERIAL AND METHODS}

\section{Patient Population}

Between March 1990 and December 2003, 42 patients (11 men and 31 women) with glomus jugulare tumors underwent GKS at our center. Twenty-three patients $(55 \%)$ had undergone one or more previous surgeries (range one-three); the mean time from the last surgery to GKS was 62.4 months (range 2-158 months). In 20 of these patients tumor growth was documented before the procedure. Nineteen patients $(45 \%)$ underwent GKS as their primary tumor management. Three of these patients had growing tumors, whereas 16 patients underwent GKS after a diagnosis was established. 
The patient characteristics are outlined in Table 1. Of note, patients in whom primary GKS was performed were older (mean age 66.7 years compared with 51.8 years, $\mathrm{p}<$ $0.01)$. Patients who underwent prior surgery were more likely to experience facial weakness and to be deaf $(48 \%$ compared with $11 \%, \mathrm{p}=0.02$ in both instances).

\section{Radiosurgical Dosimetry}

Radiosurgery was performed using the Leksell Gamma Knife (Elekta Instruments, Norcross, GA) Model U was used before March 1997, and thereafter, the procedure was performed using model B. Stereotactic MR imaging was the modality used for dose planning in 41 patients; in the other one planning was aided by cerebral angiography. Multiple-shot dose plans were created to cover conformally the often irregularly shaped tumors. The mean number of isocenters used was 8.3 (range 3-15). Thirty-two patients $(76 \%)$ were treated at the $50 \%$ isodose line, one was treated at the $45 \%$ isodose line, and nine were treated at the $40 \%$ isodose line. The median prescription isodose volume was $13.2 \mathrm{~cm}^{3}$ (range $1.2-32.2 \mathrm{~cm}^{3}$ ). The mean tumor margin dose was 14.9 Gy (range 12-18 Gy). The mean maximum tumor dose was 31.2 Gy (range 24-37.5 Gy). There was no difference in the number of isocenters, tumor volume, and minimum or maximum radiation doses between the group of patients who had undergone previous surgery and the group undergoing primary GKS.

\section{Follow-Up Review}

Gamma knife surgery has been performed as an outpatient procedure since 1997. After GKS, all patients underwent follow-up evaluation and neuroimaging performed at $6,12,24$, and 48 months, then twice a year after GKS. The tumor diameter was ascertained in the $\mathrm{x}, \mathrm{y}$, and $\mathrm{z}$ planes and compared with studies obtained on the day GKS was performed. Tumor size was classified as unchanged, decreased, or increased. Tumor reduction was defined as a decrease in tumor size of more than $2 \mathrm{~mm}$. Conversely, tumor enlargement of more than $2 \mathrm{~mm}$ was considered progression. Tumors with less than a $2-\mathrm{mm}$ change in size were labeled as unchanged. In addition, the

TABLE 1

Characteristics in 42 patients who underwent GKS for glomus jugulare tumors*

\begin{tabular}{lccc}
\hline \hline \multicolumn{1}{c}{ Factor } & Prior Op & Primary GKS & p Value \\
\hline no. of patients & 23 & 19 & \\
M/F & $6: 17$ & $5: 14$ & 1.00 \\
mean age (yrs) & 51.8 & 66.7 & 0.002 \\
\% w/ & & & \\
impaired facial movement & 48 & 11 & 0.02 \\
impaired facial sensation & 17 & 5 & 0.47 \\
impaired hearing & 78 & 74 & 1.00 \\
deafness & 48 & 11 & 0.02 \\
9th \& 10th CN deficit & 74 & 53 & 0.27 \\
tongue weakness & 39 & 53 & 0.57 \\
headache & 4 & 11 & 0.86 \\
tinnitus & 0 & 21 & 0.07 \\
dizziness & 13 & 21 & 0.78 \\
bruit & 13 & 16 & 1.00 \\
\hline
\end{tabular}

$* \mathrm{CN}=$ cranial nerve. follow-up MR images were reviewed for evidence of adverse radiation-related effects.

Thirty-nine patients were treated more than 6 months ago and have been prospectively followed. No patient has been lost to follow-up review. The mean follow-up duration after GKS was 44 months (range 6-149 months).

\section{RESULTS}

After GKS 12 tumors (31\%) shrank (Fig. 1), 26 (67\%) were unchanged, and one tumor (2\%) enlarged. No correlation was found between tumor shrinkage and tumor margin dose $(p=0.55)$, maximum radiation dose $(p=0.4)$, or length of follow up $(p=0.66)$. The patient with tumor growth initially presented with lower cranial nerve deficits and underwent primary GKS for a lesion measuring 39 $\mathrm{mm}$ in its largest dimension (Fig. 2). The prescription isodose volume was $29.7 \mathrm{~cm}^{3}$. The tumor margin dose was 12 Gy and the maximum dose was 24 Gy. Ninety-nine months after radiosurgery, this patient experienced hearing loss, and repeated MR imaging revealed that the tumor was larger. The patient underwent repeated GKS because of his advanced age (76 years). The volume covered was $50.3 \mathrm{~cm}^{3}$, the tumor margin dose was $14 \mathrm{~Gy}$, and the maximum dose was $35 \mathrm{~Gy}$. One year after the repeated procedure the patient experienced a vocal cord paralysis, but he has not required any additional surgery to manage this complication. Follow-up MR imaging demonstrated that the tumor was unchanged in size, but edema was observed in the adjacent cerebellum. Three patients died of unrelated causes after GKS. The progression-free survival rate after GKS was $100 \%$ at 3 and 7 years, and $75 \%$ at 10 years (Fig. 3).

\section{Posttreatment Complications}

In addition to the patient with hearing loss and vocal cord paralysis described earlier, five others had complications related to the procedure. One patient had persistent headache, nausea, and vomiting in the days after GKS and required a short course of antiemetic and corticosteroid medications. One patient had increased imbalance and vertigo 9 months post-GKS, but the symptoms improved with vestibular training. One patient experienced decreased facial sensation 6 months after GKS.

The most common complication was hearing loss. Overall, five (19\%) of 26 patients with intact hearing before GKS had a subjective decline in hearing after the operation. Hearing preservation was achieved in 86 and $81 \%$ at 1 and 4 years, respectively (Fig. 4). One patient with a hormone-secreting tumor attained normal biochemical status 42 months post-GKS. The remaining patients $(82 \%)$ remained stable or improved subjectively after GKS.

\section{DISCUSSION}

The management goals for glomus jugulare tumors must take two facts into consideration. One is that, although malignant paragangliomas do occur, the majority of patients have benign tumors that grow very slowly. In fact, patients have been reported who have survived more than 40 years with no treatment. ${ }^{17}$ The other fact is that the 


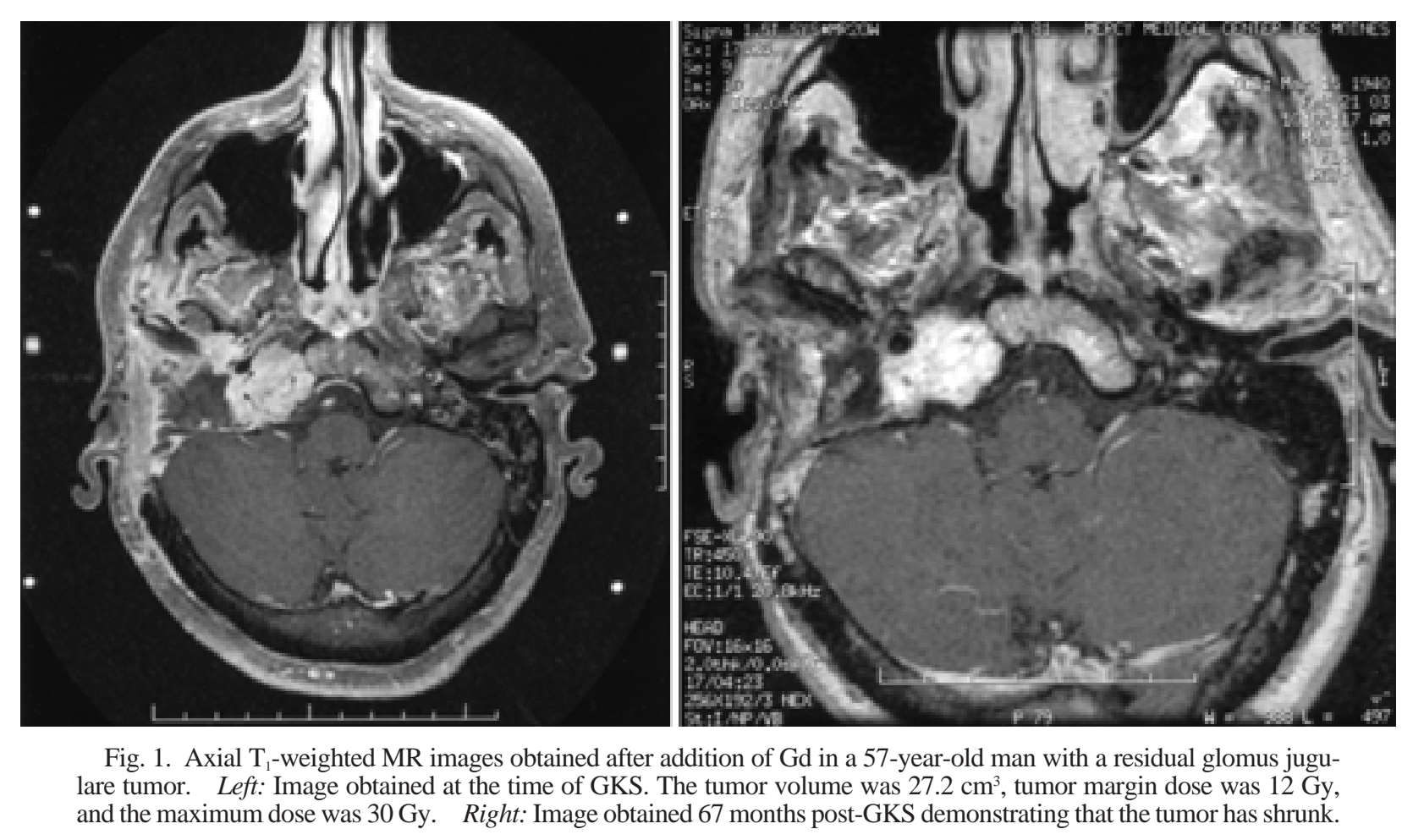

functional consequences of a lower cranial nerve injury are far more significant than injury to other cranial nerves. Ojemann ${ }^{14}$ stated in an editorial on skull base surgery that if the patient has a high probability of being cured of the tumor or of having a useful life significantly lengthened or more serious disabilities relieved, then the loss of facial nerve function or hearing is acceptable. On the other hand, the loss of ninth and 10th cranial nerve function is one of the most serious neurological disabilities for a patient. There is a real question as to whether one should electively give this disability to a patient. ${ }^{14}$ A paper by Netterville and Civantos ${ }^{13}$ on rehabilitation from glossopharyngeal, vagus, and accessory nerve deficits after neurootological skull base surgery affirmed the significance of such deficits. They said that ". . . the time required to return to a reasonably enjoyable diet often extends to one year postoperatively. A few never attain the goal of enjoyable intake and continue to struggle to maintain adequate nutrition. The latter situation is the rule, not the exception, in the elderly population." Steinberg and Holz ${ }^{17}$ also discuss this problem. Consequently, the ideal treatment for these patients should provide protection from tumor progression and a minimal chance of injury to the lower cranial nerves.

In our experience, GKS is effective in patients with glomus jugulare tumors. At a follow-up interval that extended more than 12 years for some patients, only one had tumor progression. The time to treatment failure in that patient was 99 months. Based on this case, it could be argued that GKS may slow the growth of these tumors, but after an interval they will again become active and begin to enlarge. This same argument has been advanced regarding the use of fractionated radiation therapy for glomus jugulare tumors. ${ }^{1}$ Nevertheless, almost one third of our patients had a reduction in tumor size after GKS.
Another criticism could be that for patients in whom there was no reduction in tumor size after GKS, we are simply seeing the natural history of these tumors, which may remain quiescent for decades even without treatment. Nevertheless, in 23 (59\%) of 39 patients in whom evaluation was possible, tumor growth was documented on serial imaging before GKS was performed.

It should be noted that the tumor margin dose for our patient who failed GKS was 12 Gy. Therefore, higher radiation doses to the tumor margin may be required to provide long-term growth control. We intend to follow patients closely when they undergo GKS for benign tumors so that we can clearly define the success of this procedure, and we will evaluate whether reduced doses are associated with higher rates of tumor progression at extended follow-up intervals. ${ }^{8}$ An extended follow-up period is also necessary to understand better the incidence of radiationinduced neoplasms after GKS. ${ }^{12}$

Gamma knife surgery has been proven to be safe for patients with glomus jugulare tumors. New permanent neurological deficits occurred in only five (13\%) of 39 patients; the most common complication was hearing loss. In our patients, the actuarial rate of hearing preservation exceeded $80 \% 4$ years post-GKS. The most likely explanation for hearing loss is not radiation injury to the vestibulocochlear nerve, but rather radiation injury to the cochlea and other temporal bone structures. ${ }^{10}$ Support for this concept comes from the fact that no patient suffered a facial weakness in our series. Increased awareness of the possibility of hearing decline related to temporal bone radiation exposure may increase the rate of hearing preservation in these patients. Nonetheless, in some patients tumors are intimately involved with the hearing apparatus and sparing these structures may not be possible if complete radiation coverage of the tumor is desired. 

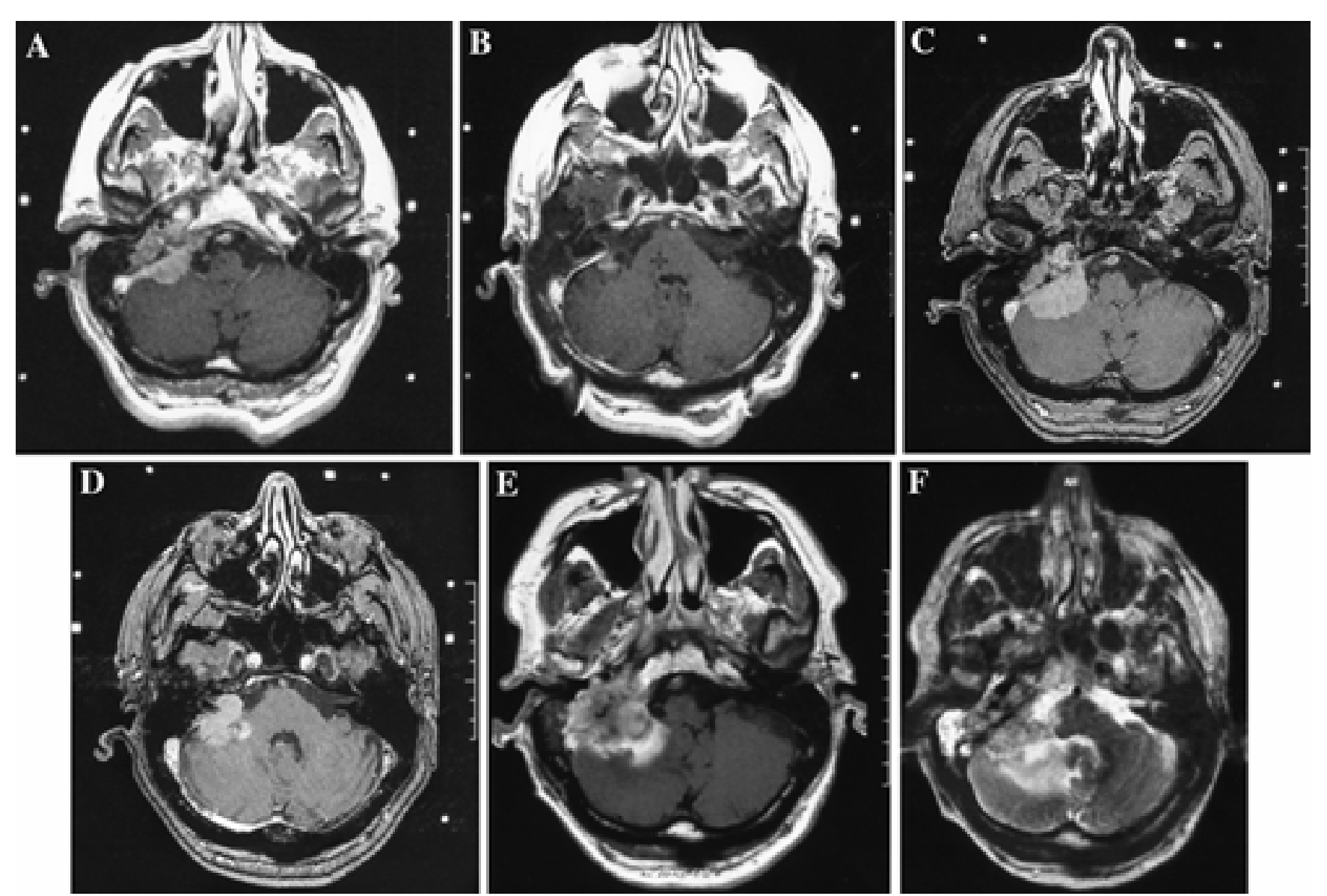

Fig. 2. A and B: Axial $\mathrm{T}_{1}$-weighted MR images obtained after addition of $\mathrm{Gd}$ in a 68 -year-old man who underwent primary GKS for a glomus jugulare tumor. The tumor volume was $29.7 \mathrm{~cm}^{3}$, tumor margin dose was $12 \mathrm{~Gy}$, and the maximum dose was $24 \mathrm{~Gy}$. C and D: An MR image obtained 101 months later at the time of repeated GKS. Note the tumor enlargement superiorly, extending into the internal auditory canal. The tumor volume was $50.3 \mathrm{~cm} 3$, tumor margin dose was $14 \mathrm{~Gy}$, and the maximum dose was $35 \mathrm{~Gy}$. E and F: An MR image obtained 1 year after repeated GKS. Note the enhancement and edema in the right cerebellar hemisphere.

Perhaps the most significant finding has been that the risk of lower cranial nerve injury is quite low. In our experience with patients who have glomus jugulare tumors and other lesions located in and around the jugular foramen,

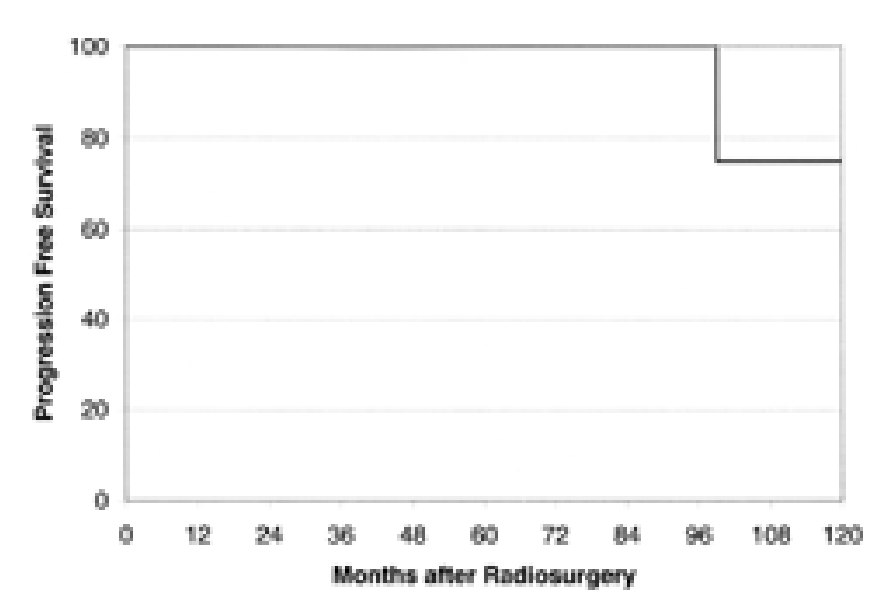

Fig. 3. Graphs showing progression-free survival after GKS in glomus jugulare tumors. none has suffered a lower cranial nerve deficit after a single session of GKS. The patient in our series who underwent two procedures experienced vocal cord paralysis 1 year after repeated GKS. As a result, we believe that it is

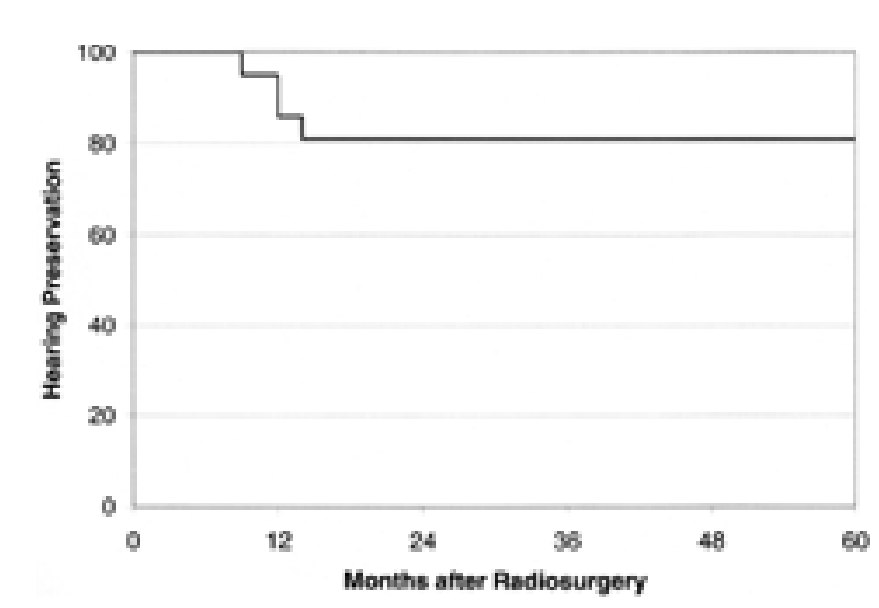

Fig. 4. Graphs showing hearing preservation after GKS in glomus jugulare tumors. 
possible that even cranial nerves thought to be relatively resistant to injury from radiation can be affected by repeated, high-dose exposures.

\section{CONCLUSIONS}

Gamma knife surgery provided tumor control with a low risk of new cranial nerve injury according to early follow-up evaluations. Radiosurgery can be safely used as a primary management tool in patients with glomus jugulare tumors that do not have significant cervical extension, or in patients who have recurrent tumors in this location.

\section{References}

1. Brackmann DE, House WF, Terry R, et al: Glomus jugulare tumors: effect of irradiation. Trans Am Acad Ophthalmol Otolaryngol 76:1423-1431, 1972

2. Cole JM, Beiler D: Long-term results of treatment for glomus jugulare and glomus vagale tumors with radiotherapy. Laryngoscope 104:1461-1465, 1994

3. Eustacchio S, Leber K, Trummer M, et al: Gamma knife radiosurgery for glomus jugulare tumours. Acta Neurochir 141: 811-818, 1999

4. Foote RL, Pollock BE, Gorman DA, et al: Glomus jugulare tumor: tumor control and complications after stereotactic radiosurgery. Head Neck 24:332-339, 2002

5. Green JD Jr, Brackmann DE, Nguyen CD, et al: Surgical management of previously untreated glomus jugulare tumors. Laryngoscope 104:917-921, 1994

6. Gstoettner W, Matula C, Hamzavi J, et al: Long-term results of different treatment modalities in 37 patients with glomus jugulare tumors. Eur Arch Otorhinolaryngol 256:351-355, 1999

7. Jordan JA, Roland PS, McManus C, et al: Stereotactic radio- surgery for glomus jugulare tumors. Laryngoscope 110:35-38, 2000

8. Kondziolka D, Nathoo N, Flickinger JC, et al: Long-term results after radiosurgery for benign intracranial tumors. Neurosurgery 53:815-822, 2003

9. Larner JM, Hahn SS, Spaulding CA, et al: Glomus jugulare tumors. Long-term control by radiation therapy. Cancer 69: 1813-1817, 1992

10. Linskey ME, Johnstone PA, O'Leary M, et al: Radiation exposure of the normal temporal bone structures during stereotactically guided gamma knife surgery for vestibular schwannomas. J Neurosurg 98:800-806, 2003

11. Liscak R, Vladyka V, Wowra B, et al: Gamma knife radiosurgery of the glomus jugulare tumour-early multicentre experience. Acta Neurochir 141:1141-1146, 1999

12. Loeffler JS, Niemierko A, Chapman PH: Second tumors after radiosurgery: tip of the iceberg or a bump in the road? Neurosurgery 52:1436-1442, 2003

13. Netterville JL, Civantos FJ: Rehabilitation of cranial nerve deficits after neurotologic skull base surgery. Laryngoscope $\mathbf{1 0 3}$ (Suppl 60):45-54, 1993

14. Ojemann RG: Skull-base surgery: a perspective. J Neurosurg 76:569-570, 1992

15. Schild SE, Foote RL, Buskirk SJ, et al: Results of radiotherapy for chemodectomas. Mayo Clin Proc 67:537-540, 1992

16. Skolyszewski J, Korzeniowski S, Pszon J: Results of radiotherapy in chemodectoma of the temporal bone. Acta Oncol 30: 847-849, 1991

17. Steinberg N, Holz WG: Glomus jugularis tumors. Arch Otolaryngol 82:387-394, 1965

Manuscript received June 15, 2004.

Accepted in final form July 14, 2004.

Address reprint requests to: Bruce E. Pollock, M.D., Department of Neurological Surgery, Mayo Clinic, 200 First Street SW, Rochester, Minnesota 55905. email: pollock.bruce@mayo.edu. 